\title{
Hyposecretion of $\beta$-Adrenergically Induced Sweating in Cystic Fibrosis Heterozygotes
}

\author{
J. K. BHHM, (i. HAGIWARA. ․ J. IFWISION. P. M. QLINTON. AND J. J. HINT:

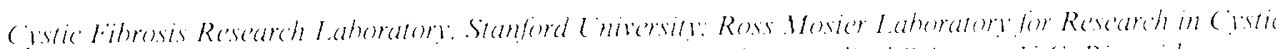

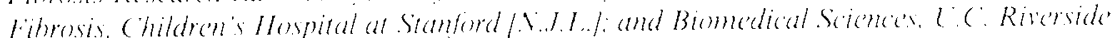 \\ Riverside /P.11.Q.). (alifornies
}

\begin{abstract}
ABSTRACI. In order to determine if expression of the cystic fibrosis gene can be detected in heterorygotes, we determined sweat responses induced by local stimulation with cholinergic and $\beta$-adrenergic agents for 20 heterozygotes, 19 age- and sex-matched controls, and five subjects with cystic fibrosis. Active sweat glands were counted and sweat droplets were collected in constant bore capillaries and measured optically. Each subject was tested two to six times. The central finding was that the sweat response of carriers was significantly lower than controls to $\beta$-adrenergic stimulation $(p=0.0013$, two-tailed $t$ test $p<0.02$, Mam-Whitney U), while cystic fibrosis homozygotes did not sweat at all. In contrast, the cholinergic sweat responses did not differ between carriers and controls. For both groups the correlation between cholinergic and $\beta$ adrenergic sweating was positive, but a linear regression of $\beta$-adrenergic sweat responses as a function of cholinergic sweat responses yielded slopes that were significantly different for the two groups. The ratio of $\beta$-adrenergic to cholinergic sweating was plotted for each subject; the mean ratio of the carriers was approximately half of the mean for the controls $(p=0.0002$ using $t$ test or $p<0.002$ using the Mann-Whitney (). Our results confirm previous studies and provide new evidence that carriers have, on average, a $\beta$-adrenergically stimulated secretory response that is significantly reduced relative to the control response. ( $P e-$ diatr Res 22: 271-276, 1987)
\end{abstract}

\section{Abbreviation}

CF, cystic fibrosis

Fecrine sweat glands are the most accessible organs affected by (1). They have thus played a key role in research aimed at identifying the basic defect. The high concentration of $\mathrm{Na}$ and (1) in the sweat of (1: homorygotes (1). which is the single most reliable indicator of the discase. results from impermeahility to (1) in the reabsorptive duct (2.3). Sinec (1) impermeability alse occurs in respiratory tissue (4), it may be a direce consecpuence of the genctic abnormality that causes ( 1 .

Until recently the secretory process of the sweat gland was thought to be normal in (1: $(5)$. Primary sweat of ( 1 homozygotes has a normal ion content (6). and secretory rates of ( $F$ and control subjects are equivalent (3, 5.7). However. while sweat

Recoived Julw ?1. 1986: accepted April 2. 1987

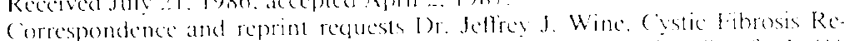
search Jabonatory Bldge. 4?0). Jordan Mall. Stanford I Iniversity. Stanford. (1) 94305

Supported by (estic Fibrosis Rescarch. Inc.. the Amy Bienensteck (istic

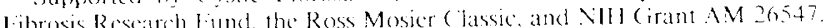

rates in response to cholinergic stimulation are normal. ( $\mathrm{H}$ : homorygotes do not sweat at all in response to ridtencrgic agonists. even though $\mathrm{AMP}$ levels within the sweat gland cells rise normally (8). These remarkable results suggest a highly specific defect in a distal stage of the excitation-secretion coupling mechanism that is engaged by s-adrenergic agonists in ( 1 sweat glands.

Sato and Sato (8) also reported that CF heteregegeres swcat less than controls in response to b-adrenergic agonists, and although no test of significance was given. the response appeared to be substantially lower than control values. We consider it especially important to reassess the cridence that heterorggotes express a defect in badrenergic swat secretion. Vo consistent phisiological correlate of heterorgesity for the (F gene has ever been established. Fen the most reliable phesiological measures that distinguish between ( $\mathrm{F}$ homorgotes and controls. namely sweat electrolyte kevels (9) and nasal transmecosal potential differences (4.10). do not detect differences between ( I: heterosgotes and controls. This may mean that the measured properties can be maintained in the normal range by decreased levels of a gene product. or it may mean that the quantity of the geme product is kept at normal levels within the heterosgotic cell by homeostatic regulatory mechanisms. If the latter is true. no test of cell proteins or the functions that depend on them will detect (Fi heterozgotes. Detection of a consistent heterorygote difference would rule out the latter possibility.

Although previous attempts to distinguish ( I heterorgetes have been inconsistent, the i-adrenergic sweating results reported by Sato and Sato (8) provide a much stronger point of departuse than results of any previous study. Their results uniquely combine wo key features: they were obtained from exocrine tissue which is known to express the defective gene, and the differences between (F" homorgotes and controls were absolute

\section{MEIHOISS}

Subjects. The $4+$ subjects comprised 20 parents of chibdren with ( $\mathrm{F}$ : (obligate heteromgotes). 19) age- and sex-matched controls. and five subjects with (1: hemorgetes. identitied by the usual criteria). One control subject was of tsian ancestes and one was black: all other subjects were (aucasian. ( 1 subjects were registered at the (estie Fibrosis (enter. ( hilden's lonpital at Stanford. I ack of sweating to s-adrencrgic agents be ( 1 . homorgotes was clearly established by Sato and Sato (8): since the effect is virtually absolute. we tested only lise subjects with repeat tests of only one. (Ad ditional characteristics of sthjects are summarized in lable 1. Because many of these subjects were known to the investigators. it was not possible to do the study blind.) Subjects were tested Januar--June with each group represented throughout. Procedures were approved by the Stanford Medical Committee for the llse of Human Subjects in Research: informed consent was obtained from cach subject. 
Table 1. Subject characteristics

\begin{tabular}{lrccc} 
& $n$ & Age \pm SEM & Asthma $(n)$ & Atopy* $^{*}(n)$ \\
\hline Control males & 11 & $37.7 \pm 2.4$ & 1 & 3 \\
Heterozygous males & 12 & $40.6 \pm 2.5$ & 1 & 2 \\
Control females & 8 & $38.2 \pm 2.7$ & 0 & 1 \\
Heterozygous females & 8 & $40.4 \pm 4.2$ & 1 & 5 \\
CF males & 4 & $31.8 \pm 3.8$ & 0 & 1 \\
CF female & 1 & 25.0 & 0 & 1 \\
\hline
\end{tabular}

* Number of subjects who had previously been tested for allergies.

Drug delivery and sweat collections. Intradermal Stimulation by $\beta$-Agonists. We used modifications of methods reported previously (8). Sweating was induced on the volar surface of the forearm by an intradermal injection of $0.2 \mathrm{ml}$ of a mixture of 8 $\times 10^{-5} \mathrm{M}$ isoproterenol, $10^{-2} \mathrm{M}$ theophylline (as aminophylline) and $1.4 \times 10^{-4} \mathrm{M}$ atropine. This combination of a $\beta$-agonist, phosphodiesterase inhibitor, and muscarinic cholinergic blocker was previously shown to elicit a pure $\beta$-adrenergic sweat response, as indicated by its total block by propranolol (1). We tested one control and one heterozygote with propranolol, and also obtained complete block of the response (data not shown). After the area was rubbed with Sylgard 184 (Dow Corning), a well was placed over the $7-\mathrm{mm}$ wheal and water-saturated mineral oil immediately added. This procedure was performed on all heterozygotes and controls from two to six times (mean measures per subject $=3.9 \pm 0.19 \mathrm{SEM}$ for this and all subsequent numbers). In about one-fifth of the sessions, the above procedure was carried out in duplicate at separate sites on the forearm (see below).

Iontophoretic Stimulation by Cholinergic Agents. This procedure was adapted from a method previously used by Bijman and Quinton (3). A current of $200 \mu \mathrm{A}$ was passed for 5 min through a solution of $2 \%$ acetylcholine and $0.1 \%$ methacholine in a pipette (tip diameter $1 \mathrm{~mm}$ ) pressed against the volar surface of the forearm. An identical well was then fixed to the arm so the site of stimulation was barely out of the field of view, and watersaturated mineral oil was immediately applied. This procedure was performed on each subject from one to seven times (mean $3.3 \pm 0.26)$.

The proximal-distal position of cholinergic and $\beta$-adrenergic stimulation was alternated for each session for each subject; when two $\beta$-agonist responses were measured, the cholinergic stimulation site was between them. Each stimulation site was at least $4 \mathrm{~cm}$ from any other.

Collection of Sweat. Before each collection the number of secreting sweat glands was counted in a $12.6 \mathrm{~mm}^{2}$ area defined by a grid in a stereomicroscope at $\times 25$. After counting, the droplets were drawn into constant bore $(62 \mu \mathrm{m})$ capillaries with a hand-held mouth pipet; droplets from all secreting glands were collected together with no attempt to determine individual differences among glands. Volumes were determined by measuring the lengths of the fluid columns using a micrometer at $\times 12$ (Fig. 1). Average volumes per gland are reported.

The protocols used to collect sweat differed significantly for the two kinds of stimulation. The protocols were designed to give the most reproducible results in each case, but they have the drawback that comparisons of sweat responses are strictly relative to our procedures: absolute sweat rates, including maximal rates, were not measured. For $\beta$-adrenergic stimulation, sweat was collected once, $40 \mathrm{~min}$ after stimulation, to maximize the amount of sweat obtained in each test (most sweating to $\beta$-adrenergic stimulation occurs in the first 20 min following an injection, and is virtually complete after $30 \mathrm{~min}$ ) (8).

For cholinergic stimulation, sweat rates are sometimes so fast that fusion of sweat droplets from adjacent glands can occur, making accurate counts of active glands impossible. To minimize this problem, all sweat produced in the first $10 \mathrm{~min}$ by cholinergic stimulation was discarded; then glands were counted and sweat collected after each of the next two 5-min intervals. There was
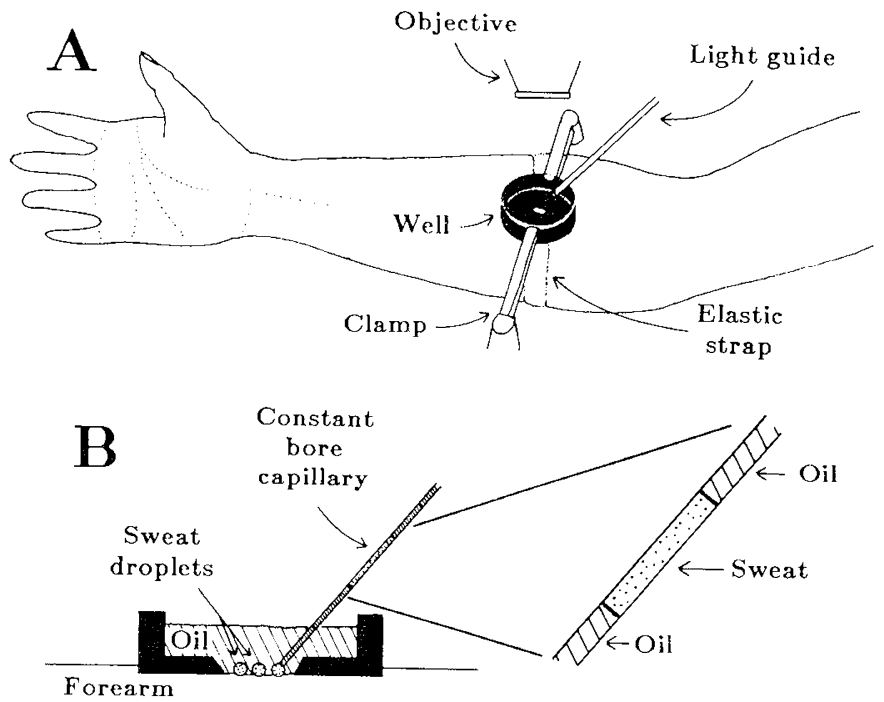

Fig. 1. Experimental arrangement. $A$, oil-filled plastic wells are secured to the arm with elastic straps, and then the arm and well are held securely to the table by a metal clamp, positioned so that the hole in the center of the well can be viewed through the microscope. Only one well is shown, but as many as three were attached, and then sequentially clamped for viewing and sweat collection. $B$, Schematic view of the method for collecting and measuring sweat droplets (4).

no systematic difference in sweat responses between the two collections.

Estimation of number of tests required. In order to estimate the number of tests required to give a reasonably accurate estimate of each subject's $\beta$-adrenergic and cholinergic sweat responses, we initially tested 12 subjects on each of 5 separate days. An analysis of the results indicated that day-to-day variations in cholinergic and $\beta$-adrenergic responsiveness within subjects were uncorrelated and that just two tests of cholinergic responsiveness and three tests of $\beta$-adrenergic responsiveness were sufficient to give mean ratios for the heterozygote and control groups that were essentially equal to the mean ratios obtained from all five tests. (Mean results for each individual shifted by at most $\pm 6.5 \%$ of their original value.) Accordingly, most remaining subjects were given two cholinergic and three to four $\beta$-adrenergic tests. In summary, for each subject each of the following measures was obtained for (on average) three to four different tests: 1 . number of glands in $12.6 \mathrm{~mm}^{2}$ secreting in response to acetylcholine; 2 . number of glands in $12.6 \mathrm{~mm}^{2}$ secreting in response to isoproterenol; 3 . volume of cholinergically induced sweat (as defined above); and, 4. volume of $\beta$ adrenergically induced sweat (as defined above).

These basic data were then analyzed in a variety of ways that are described in "Results." Significance was tested using both Student's $t$ test and the nonparametric Mann-Whitney U test (11), which does not require that the data be normally distributed.

Adverse reactions. Two of the 44 subjects tested, both control males with strong allergic histories, experienced mild delayed hypersensitivity ( $48 \mathrm{~h}$ ) skin reactions at the sites of the $\beta$-adrenergic injections. Both of these responded well to local application of corticosteroid cream. Several other subjects gave strong allergic histories but did not experience any side effects of the testing.

\section{RESULTS}

Sweating by heterozygotes in response to $\beta$-adrenergic stimulation is significantly reduced relative to a matched control sample. The central finding is that heterozygotes' sweat responses are significantly lower than control responses to $\beta$-adrenergic stimulation ( $p=0.0013$, two-tailed $t$ test; $p<0.02$, Mann-Whitney $\mathrm{U}$ ), while homozygotes do not sweat at all (difference from 
control group significant at $p<0.00(0) 1)$. For heterozygotes versus controls, a significant difference was maintained for males compared separately (Fig. $2, p=(0.0(0) 34)$, and for females $(p=0.046)$. In contrast, the cholinergic sweat responses did not differ between heterozygotes and controls. The cholinergic sweat responses of the ('l males were significantly lower than controls $(p=0 .(0)(292)$. probably because of the reduced activity among the (F males. all of whom were hospitalized at the time of their tests. These findings agree with and extend the results of Sato and Sato (8).

Sources of variahility: Responses to cholinergic agents vary greatly among individuals. Among the factors that contribute to variability in normal sweat responses are age, gender, activity level, race, and seasonal changes (12,13). Previous data suggested that cholinergic and $\beta$-adrenergic sweat responses might be positively correlated (8). We therefore asked: To what extent are sweat responses to $\beta$-adrenergic agonists consistent for an individual, what is the distribution of sweat responses to the two kinds of stimulation within the heterozygote and control populations, and what is the relation between cholinergically induced and $\beta$-adrenergically induced sweat responses?

To assess these variables a joint plot was made of the mean \pm

A

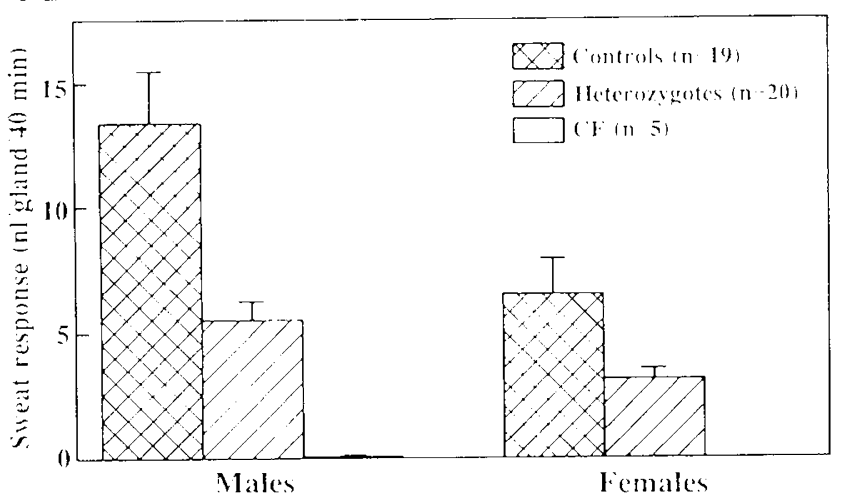

SEM of cholinergic and p-adrenergic sweat responses for cach subject. and the linear regression of b-adrenergic sweating on cholinergic sweating was calculated for heterosygotes and controls. The results are shown in Figure 3. Fach point in Figure? represents the mean value for an individual subject. The error bars show that most subjects were quite consistent, but there was large intersubject variability.

For both controls and heterozygotes the correlation between cholinergic and b-adrenergic sweating was positive: the correlation coeflicients were 0.59 and 0.67 . respectively. A linear regression of ib-adrenergic sweating as a function of cholinergic swating vielded slopes that were significantly different for the two groups: for controls the stope of 3 -adrenergic sweating as a function of cholinergic sweating was (0.187. While for heterosygotes the slope was only 0.064 . This difference is significant. $l \cdot(1.35)=25.6 . p<0.001$

The shallow slope for heterorggotes means that the separation between heterozygotes and controls was much greater at the higher cholinergic sweat responses. and this in turn means that males, who typically sweat more to cholinergic stimuli than females, will usually be easier to differentiate with this test.

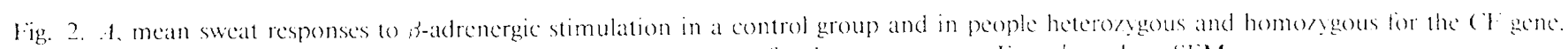
divided according to sex. B. mean values for cholinergic sweat responses for the same groups. From hom show SI: 11.

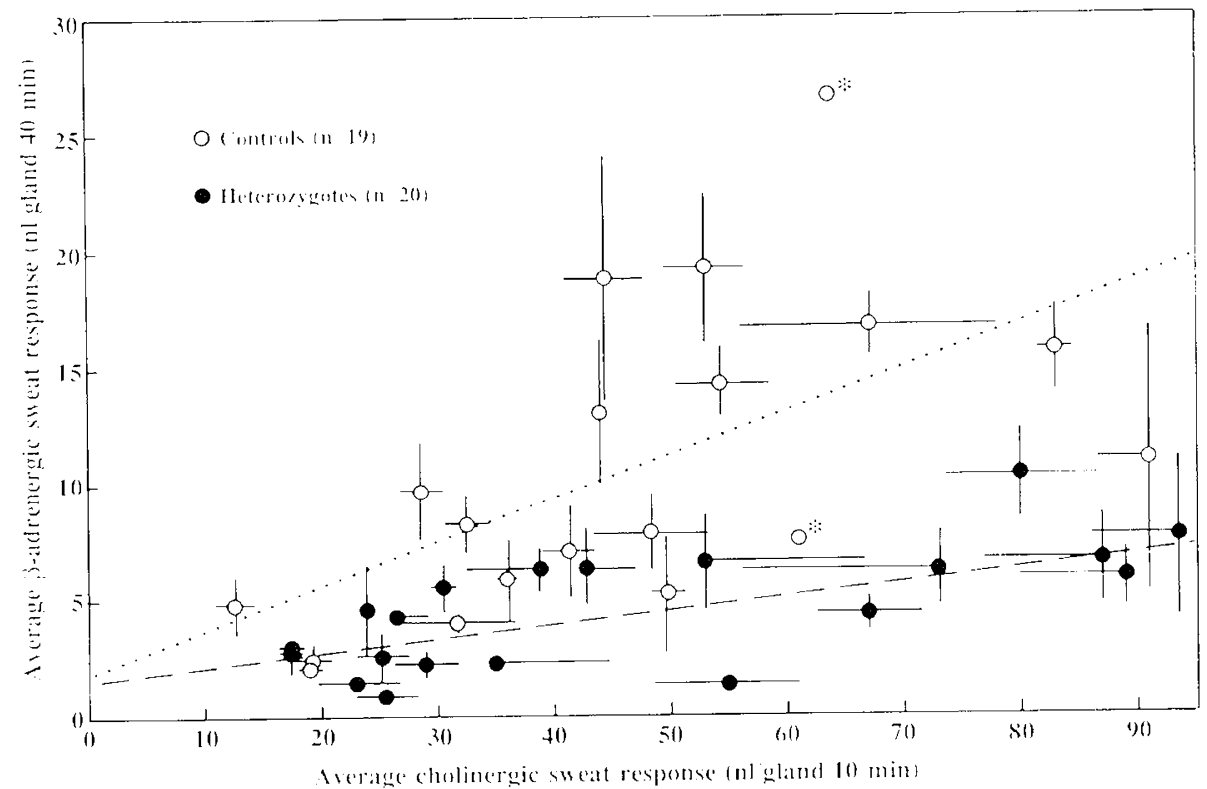

Fig. 3. B-adrenergically induced sweat responses (ordinate) plotted as a function of cholinergically induced sweat responses. Fate poin is the mean of two to six p-adrenergic tests and one to five cholinergic tests for a single individual; eror hars show St:M. fiterisk indicate two individuals who experienced contact dermatitis and therefore had only one session consisting of one cholinergic and two b-adrenergic tests. Apparent latek of error bars in other cases indicates that SIMS were less than the diameter of the rigmbol. cl homozgotes are not shown on this graph. Regresion lines shown for cach group differ significantly (see text). 
Given the positive correlation between cholinergically induced and $\beta$-adrenergically induced sweat responses, the distribution of $\beta$-adrenergic sweat responses within each group was compared by determining the ratio of $\beta$-adrenergic to cholinergic sweating for each subject, to help compensate for large individual differences in gland size and hence sweat responses. The ratio was determined by dividing the mean $\beta$-adrenergically induced sweat response by the mean cholinergically induced sweat response for each subject. The results are shown in Figure 4. Several features of these distributions are noteworthy. First, the mean $\beta$-adrenergic:cholinergic ratios were $0.23,0.11$, and 0.00 for controls, heterozygotes, and homozygotes, respectively. The heterozygote ratio differed significantly from both controls $(p=0.002)$ and homozygotes $(p=0.000)$. Second, the coefficients of variation were essentially equal $(47.9 \%$ for heterozygotes versus $48.3 \%$ for controls). Third, the use of a ratio measure eliminated the sex difference that is apparent in Figure 2. Finally, there is a sugges-

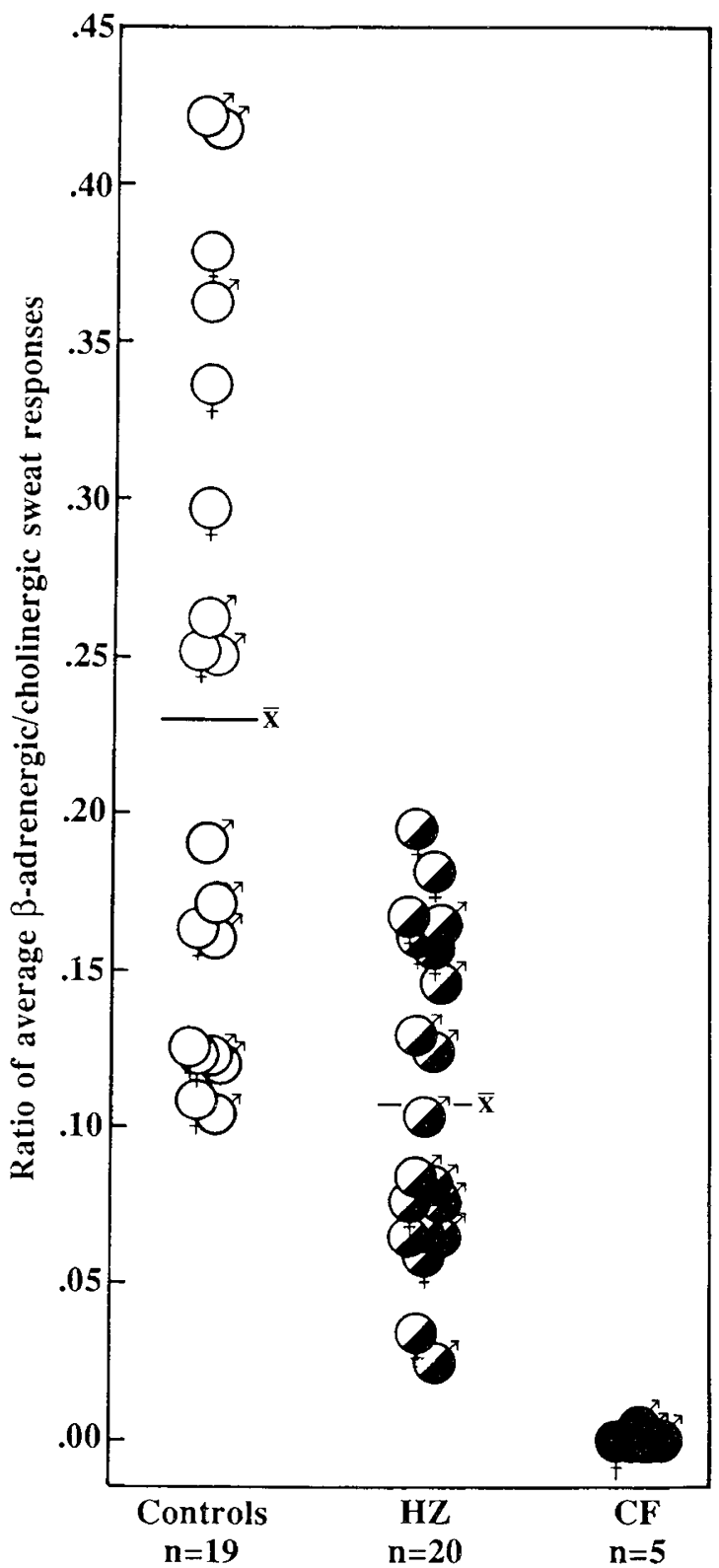

Fig. 4. Ratio of sweat responses to $\beta$-adrenergic versus cholinergic agonists for each individual, with gender indicated, grouped as controls, heterozygotes, and homozygotes. Each point was obtained by dividing the mean $\beta$-adrenergically induced sweat response for an individual by their mean cholinergically induced response. tion of bimodality in each distribution, or at least a clustering of points at the low end of each distribution, which might indicate that the nonparametric Mann-Whitney $U$ is the more appropriate test.

Active gland counts. Cholinergic Stimulation. The number of glands secreting in the $12.6 \mathrm{~mm}^{2}$ test area were counted for every test; this number was multiplied by 7.94 to give the number of glands per $\mathrm{cm}^{2}$. Results are summarized in Table 2 and Fig. 5. The gland density seen with cholinergic stimulation in our experiments was similar to that previously reported by Kawahata (14) for upper extremities of Japanese males induced to sweat physiologically (mean 183 glands per $\mathrm{cm}^{2}$ for four males aged 20-35). The number of glands induced to sweat with cholinergic stimulation did not differ between controls and heterozygotes.

Our results with cholinergic stimulation also confirmed an unusual sex difference noted in previous studies using thermal stimulation (15-17); men had significantly fewer active glands than women $(p=0.007)$. Also, there was an overall negative correlation of sweat rate with gland number $(r=-0.37, p<$ 0.05 ).

For females, the mean number of glands per $\mathrm{cm}^{2}$ was 152 and the mean sweat response was $26.9 \mathrm{nl} /$ gland/10 min, to give a mean sweat response of $4.09 \mu \mathrm{l} / \mathrm{cm}^{2} / 10 \mathrm{~min}$, while for males the equivalent figures were 118 glands, $60.1 \mathrm{nl} /$ gland $/ 10 \mathrm{~min}$, and $7.09 \mu \mathrm{l} / \mathrm{cm}^{2} / 10 \mathrm{~min}$.

$\beta$-Adrenergic Stimulation. The pattern of active gland counts versus sweat responses to $\beta$-adrenergic stimulation (Fig. 6) has a different form from the plot of cholinergically induced sweating. For $\beta$-adrenergic stimulation the correlation between sweat gland number and sweat response is positive $(r=0.61, p<0.01)$. Furthermore, the sex difference that is so apparent with cholinergic stimulation is no longer seen, and, most importantly, heterozygotes have significantly fewer active glands (Table $2, p$ $=0.0015)$. The lower active gland counts could indicate that more glands are refractory to $\beta$-adrenergic stimulation in heterozygotes, or it could simply reflect the decreased secretory responses already documented.

Table 2. Mean counts of active sweat glands in $12.6 \mathrm{~mm}^{2}$ area

\begin{tabular}{lccccc} 
& \multicolumn{3}{c}{$( \pm S E M)$} & & \\
\cline { 5 - 6 } & \multicolumn{2}{c}{ Cholinergic } & & \multicolumn{2}{c}{$\beta$-adrenergic } \\
\cline { 2 - 3 } \cline { 5 - 6 } & Male & Female & & Male & Female \\
\hline Control & $15.6 \pm 1.0$ & $20.4 \pm 1.7$ & & $12.1 \pm 1.2$ & $12.7 \pm 1.1$ \\
Heterozygotes & $14.3 \pm 0.7$ & $18.0 \pm 0.8$ & & $9.5 \pm 0.5$ & $8.3 \pm 0.7^{*}$ \\
CF & $23.0 \pm 2.3$ & 22.0 & $0.2 \pm 0.2 \dagger$ & $0.5 \pm 0.0$ \\
\hline
\end{tabular}

$* p=0.008$ compared to control females.

$\dagger p=0.0000$ compared to control males.

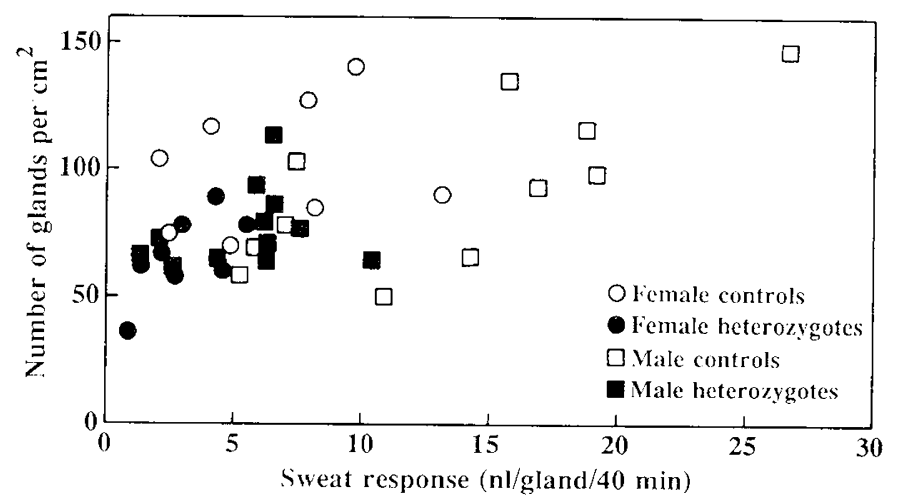

Fig. 5. Number of active glands per $\mathrm{cm}^{2}$ after cholinergic stimulation, as a function of sweat response, for heterozygotes and controls. There was no significant difference between the groups, but females had significantly more active glands and lower sweat responses than males. 


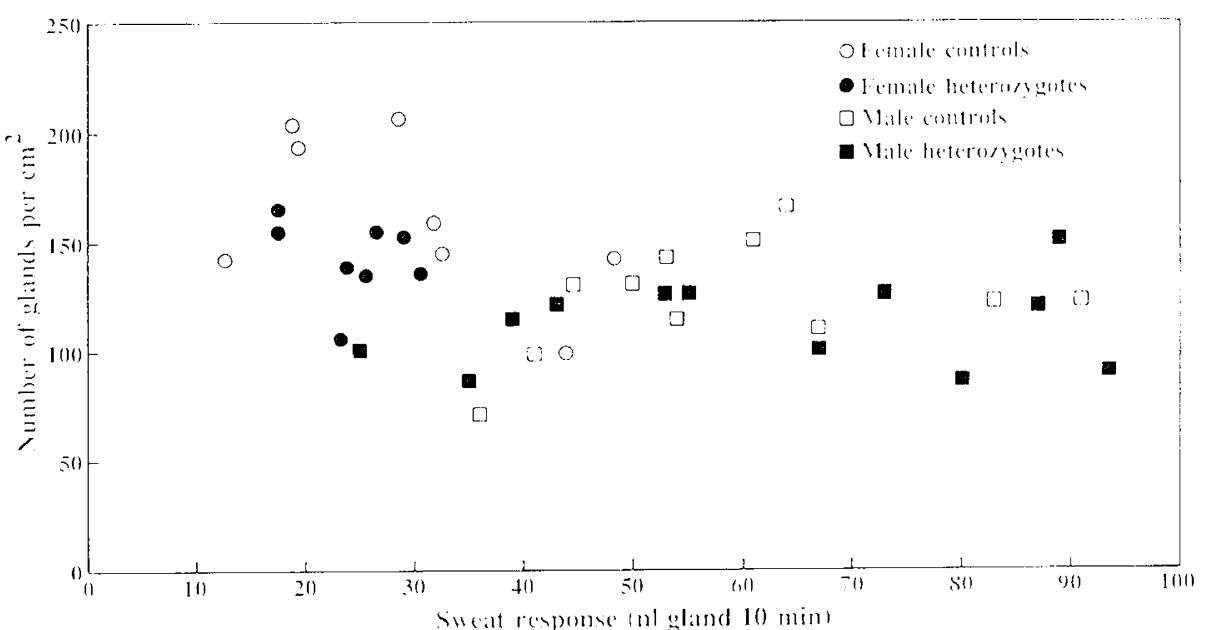

Fig. 6. Number of active glands per cme after b-adrenergic stimulation. as a function of sweat response for heterosegetes and controls. Heterosygetes had significanty fewer active glands than controls.

How can these patterns be explained? (One possibility is that chelinergic stimulation causes detectable sweating in a large and consistent proportion of glands and so reveals intrinsic differences in gland density among individuals. If that were true, the greater gland density of females might reflect their generally smaller body size. since there is evidence that gland number is fixed early in life. so that lower gland densities are expected in larger individuals. In contrast, fo-adrenergic stimulation. Which evokes a smaller secretory response. might on that basis alone be expected io cause detectable sweating in a smaller proportion of glands. In such a circumstance. the proportion of secreting glands and the apparent gland density will both be increased by factors that increase the sweat rate. and this could produce the positive correlation between the apparent gland density and sweat response that was observed (Fig. 6).

\section{I)IS( ISSION}

Our results contirm those of Sato and Sato (1) and provide new evidence that heterorygotes have, on average, a is-adrenergically stimulated secretory response that is significantly reduced relative to the control response. These data require three facts to be interpreted: Why is B-adrenergic stimulated sweating absent in (' homorygotes and reduced in heterorvgotes'? Why is cholinergically stimulated sweating unaffected in ( $T^{*}$. Why do reliable tests for (I homorygotes namely sweat chloride levels and nasal potential difference measurements. generally fail to distinguish between heterozygotes and controls?

Our explanation for the absence of B-adrenergic-stimulated sweating in ( $\mathrm{F}$ homozygotes and hyposecretion in heterozgotes begins with the general model of (1 -mediated fluid secretion developed by rrizell ("lal. (18) (Fig. 7) and since widely confirmed (19). Our hypothesis is that ib-adrenergic-stimulated sweat secretion fails to occur in ( 1 because apical (l channels in the sweat gland eells fail to open in response to the rise of cytosolic cAMP (2()). This hypothesis is based on the original lindings of defective excitation-secretion coupling but normal CAMP response in $\beta$-adrenergic-stimulated sweating by Sato and Sato (1): on evidence for normal stimulation of $\mathrm{CAMP}$ in respiratory tissue from ( 1 subjects $(21-23)$; on the inabitity of forskolin or membrane-permeable (AMP analogs to gate chloride channels from (I: tissue (20) and on growing evidenee that the basic defect in ( F involves CAMP-gated apical (Cl channels (3. 5. 20. 24).

This explanation differs from prior interpretations of autonomic defects in (r: homorygotes and heterorygotes. The reported differences include increased airway reactivity 10 methacholine, increased pupillary responsiveness to both cholinergic and r-adrencrgic stimulation, and decreased CAMP levels in

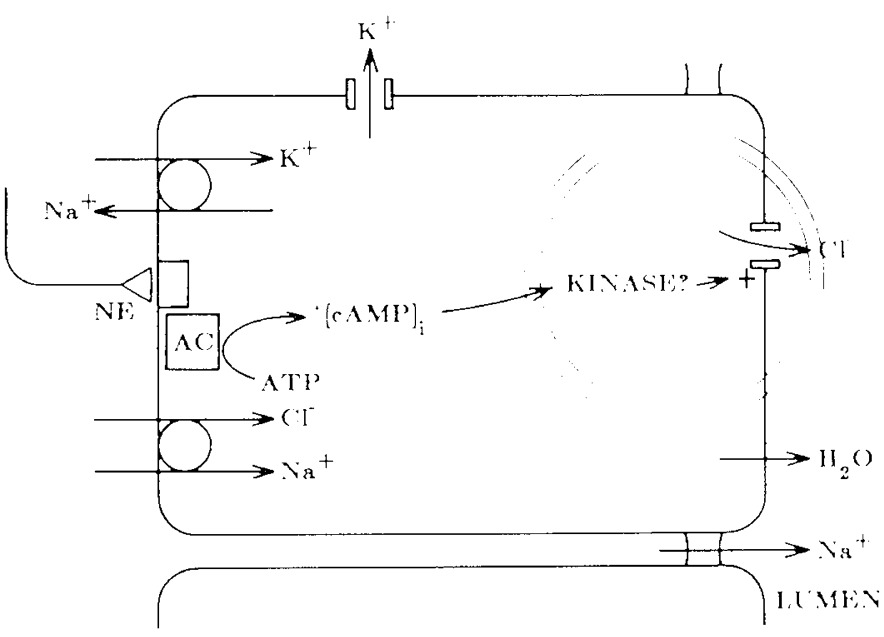

fig. 7. Simplified schematic diagram of the excitation-secretion pathway for ib-adrenergic-stimulated sweating and the site of the kefert that affects sweat secretion of ( $\mathrm{l}$ homosgotes and heterosgotes (1).

kukocrtes stimulated with isoproterenol (25-29). The premise of most of this work was that a defect exists at some carly stage of the response pathway either the b-adrenergic receptors themselves (29) or receptor-cyclase coupling (28). Although we hypothesize that the reduced s-adrenergic sweating of heterosygotes is a result of the same post-c $\triangle M P$ defect that was tirst decumented in homorgetes by Sato and Sato (1). we have not shown this directly. and in light of the persistent findings of reduced cAMP responses in leukocites of heterosgotes $(25-29)$. it will be important to determine a AMP responses to badrencrgic stimulation in sweat glands of heterorggotes.

Why do cholinergic sweat rates remain normat in (I: homozagotes? We think the eridence suggests that different mechanisms exist for sweating in response to cholinergic and is-adrenergic agents. It is clear that the carls stages of excitationsecretion differ. (holinergic sweating differs from is adrencrgic sweating in that it requires extracellular ( $a^{2}$ and is more copious (30). Cholinergic sweating might employ the basic model for secretion mentioned carlier, but with additional (a" activated pathways operating in parallel: e.s. the apical ('l channels might be gated by (a)" as well as by CAMP (20)) or (a" might gatce a basolateral $\mathrm{K}^{+}$channel or stimulate a $\mathrm{K}^{+}$pump to hyperpolaric the ecll and enhance the secretory response (31). Hewever. we 
think it is also possible that a different mechanism exists which does not rely on passive chloride flow from the cell.

If heterozygotes do express the $\mathrm{CF}$ gene, it remains to explain why tests of sweat chloride concentrations (9) and nasal potential differences $(5,10)$ have generally failed to distinguish between heterozygotes and controls. We do not yet have a quantitative model for fluid and electrolyte transport in these tissues $(32,33)$, and therefore we cannot predict the values expected for heterozygotes. However, general considerations suggest that most physiological measurements of heterozygotes will be near normal, because the gene product or the function it controls is usually present in excess, so that a $50 \%$ reduction is usually not rate limiting for the process being measured. The challenge is to move from generalizations to specifics: we recognize the importance of providing quantitative models of how decreased chloride permeability will affect higher order physiological processses in different systems, and are attempting to obtain the data needed for such models.

In summary, we propose that both the absence of sweating in $\mathrm{CF}$ homozygotes and the reduced sweating of CF heterozygotes reflect reduced levels of a gene product that is rate limiting for $\beta$-adrenergic-stimulated sweat secretion. The gene product is still unidentified, but salient possibilities are cAMP-gated chloride channels, or a molecule involved in gating those channels. Experiments with cells from heterozygotes should be especially informative in identifying the gene product. Increased availability is one advantage: CF heterozygotes are approximately 100 times more prevalent than CF homozygotes in the United States population. But beyond that, affected heterozygous cells should have distinct experimental advantages, since they can be viewed as fusion products of normal and homozygous cells.

Acknowledgments. The authors thank Jan Ruby for help with preparation of the manuscript, W. S. Farrell for advice on statistical tests, and especially our volunteer subjects, each of whom devoted many hours to this study.

\section{REFERENCES}

1. di Sant'Agnese PA, Darling RC, Perera GA, Shea E 1953 Sweat electrolyte disturbances associated with childhood pancreatic disease. Am J Med 15:777-784

2. Quinton PM 1983 Chloride impermeability in cystic fibrosis. Nature (Lond) $301: 421-422$

3. Bijman J, Quinton PM 1984 Influence of abnormal $\mathrm{Cl}^{-}$impermeability on sweating in cystic fibrosis. Am J Physiol 247: C3-C9

4. Knowles MR, Carson JL, Collier AM, Gatzy JT, Boucher, RC 1981 Measurements of nasal transepithelial electric potential differences in normal human subjects in vivo. Am Rev Respir Dis 124:484-490

5. Quinton PM 1982 Abnormalities in electrolyte sccretion in cystic fibrosis sweat glands due to decreased anion permeability. In: Quinton PM, Martinez JR. Hopfer U (eds) Fluid and Electrolyte Abnormalities in Exocrine Glands in Cystic Fibrosis. San Francisco Press, San Francisco, pp 53-76
6. Shulz IJ 1969 Micropuncture studies of the sweat formation in cystic fibrosis patients. J Clin Invest 48:1470-1477

7. Gochberg SH, Cooke RE 1956 Physiology of the sweat gland in cystic fibrosis of the pancreas. Pediatrics 18:701-715

8. Sato K, Sato F 1984 Defective beta adrenergic response of cystic fibrosis sweat glands in vivo and in vitro. $\mathrm{J}$ Clin Invest $73: 1763-1771$

9. Shwachman H, Mahmoodian A 1967 Pilocarpine iontophoresis sweat testing results of seven years' experience. Mod Probl Pediatr 10:158-182

10. Gowen CW, Lawson EE, Gingras-Leatherman J, Gatzy JT, Boucher RC, Knowles MR 1986 Increased nasal potential difference and amiloride sensitivity in neonates with cystic fibrosis. J Pediatr 108:517-521

11. Siegel S 1957 Nonparametric Statistics for the Behavioral Sciences. McGrawHill, New York

12. Kuno Y 1956 Human Perspiration. Charles C Thomas, Springfield, IL

13. Sato K, Sato F 1983 Individual variations in structure and function of human eccrine sweat gland. Am J Physiol 245:R203-R208

14. Kawahata $A 1939$ Numerical studies on the human active sweat glands. NihonSeirigaku-Zasshi 4:438-444

15. Kawahata A 1960 Sex differences in sweating. In: Yoshimura H, Ogata K, Itoh $\mathrm{S}$ (eds) Essential Problems in Climatic Physiology. Nankodo, Kyoto, Japan, pp 169-184

16. Morimoto T, Slabochova Z, Naman RK, Sargent F II 1967 Sex differences in physiological reactions to thermal stress. J Appl Physiol 22:526-532

17. Bar-Or O, Lundgren HM, Magnusson LI, Buskirk ER 1968 Distribution of heat-activated sweat glands in obese and lean men and women. Hum Biol 40:235-248.

18. Frizzell RA, Field M, Schultz SG 1979 Sodium-coupled chloride transport by epithelial cells. Am J Physiol 236:F1-F8

19. Welsh MJ 1983 Intracellular chloride activities in canine tracheal epithelium. Direct evidence for sodium-coupled intracellular chloride accumulation in a chloride-secreting epithelium. J Clin Invest 71:1392-1401

20. Frizzell RA, Rechkemmer G. Shoemaker RL 1986 Altered regulation of airway epithelial cell chloride channels in cystic fibrosis. Science $233.558-560$

21. Boucher RC, Stutts MJ, Knowles MR, Centley L, Gatzy JT $1985 \mathrm{Na}^{+}$transport in cystic fibrosis nasal epithelia: abnormal basal rate and response to adenylate cyclase activation. Clin Res 33:467A(abstr)

22. Welsh MJ, Liedtke CM 1986 Chloride and potassium channels in cystic fibrosis airway epithelia. Nature 322:467-470

23. Widdicombe JH 1986 Effects of cystic fibrosis on the $\beta$-adrenergic response of primary cultures of airway epithelia. Am J Physiol 251:R818-R822

24. Widdicombe JH, Welsh MJ, Finkbeiner WE 1985 Cystic fibrosis decreases the apical membrane chloride permeability of monolayers cultured from cells of tracheal epithelium. Proc Natl Acad Sci USA 82:6167-6171

25. Davis PB 1984 Autonomic and airway reactivity in obligate heterozygotes for cystic fibrosis. Am Rev Respir Dis 129:911-914

26. Davis PB, Braunstein M, Jay C 1978 Decreased adenosine $3^{\prime}: 5^{\prime}$-monophosphate response to isoproterenol in cystic fibrosis leukocytes. Pediatr Res 12:703-707

27. Davis PB, Shelhamer JR, Kaliner M 1980 Abnormal adrenergic and cholinergic sensitivity in cystic fibrosis. N Engl J Med 302:1453-1456

28. Davis PB, Dieckman L, Boat TF, Stern RC, Doershuk CF 1983 Beta adrenergic receptors in lymphocytes and granulocytes from patients with cystic fibrosis. J Clin Invest 71:1787-1795

29. Galant SP, Norton L, Herbst J, Wood C 1981 Impaired beta adrenergic receptor binding and function in cystic fibrosis neutrophils. J Clin Invest $68 \cdot 253-258$

30. Sato K, Sato F 1981 Role of calcium in cholinergic and adrenergic mechanisms of eccrine sweat secretion. Am J Physiol 241:C113-C120

31. Dharmsathaphorn K, Pandol SJ 1986 Mechanism of chloride secretion induced by Carbachol in a colonic epithelial cell line. J Clin Invest 77:348-354

32. Knowles M, Gatzy J, Boucher R 1983 Relative ion permeability of normal and cystic fibrosis nasal epi. 'ium. J Clin Invest 71:1410-1417

33. Quinton PM 1986 Missing Cl Jnductance in cystic fibrosis. Am J Physiol 251:C649-C652 